\title{
Qualidade da madeira serrada de cinco espécies de Eucalyptus resistentes à geada
}

\author{
Bruna Verediana Müllerr, Márcio Pereira da Rocha², Ricardo Jorge Klitzke², Alexsandro \\ Bayestorff da Cunha ${ }^{3}$, José Reinaldo Moreira da Silva ${ }^{4}$, Morgana Cristina França ${ }^{2}$, Marcos Felipe \\ Nicoletti ${ }^{3}$ \\ ${ }^{1}$ Instituto Federal de Educação, Ciência e Tecnologia Catarinense, Rio do Sul, Santa Catarina, Brasil. \\ ${ }^{2}$ Departamento de Engenharia e Tecnologia Florestal, Universidade Federal do Paraná, Curitiba, Paraná, Brasil. \\ ${ }^{3}$ Engenharia Florestal, Universidade do Estado de Santa Catarina, Lages, Santa Catarina, Brasil. \\ ${ }^{4}$ Departamento de Ciências Florestais, Universidade Federal de Lavras, Lavras, Minas Gerais, Brasil.
}

\begin{abstract}
RESUMO O cultivo de Eucalyptus no Sul do Brasil ainda ocorre de forma limitada devido à ocorrência de geadas e ao número restrito de espécies adaptadas à essa condição. Assim, este estudo objetivou avaliar a qualidade da madeira serrada de cinco espécies de Eucalyptus, cultivadas em região de ocorrência de geadas. Foram selecionadas árvores de Eucalyptus benthamii, Eucalyptus deanei, Eucalyptus dorrigoensis, Eucalyptus dunnii e Eucalyptus smithii, com 18 anos, seccionadas em toras e divididas em classes diamétricas de (1) 20,1 a $25,0 \mathrm{~cm}$; (2) 25,1 a $30,0 \mathrm{~cm}$ e (3) maior que $30,0 \mathrm{~cm}$. As toras foram desdobradas, produzindo tábuas com faces tangenciais. Antes e após a secagem natural, as intensidades de rachaduras e empenamentos (arqueamento, encurvamento e encanoamento) das tábuas foram determinadas. Os resultados obtidos indicaram que o diâmetro de toras exerceu pouca influência sobre a qualidade da madeira. $O$ processo de secagem aumentou as intensidades de rachadura e arqueamento, não sendo observada tendência quanto à intensidade de encurvamento. As menores intensidades de defeitos foram observadas para Eucalyptus dunnii e Eucalyptus deanei. No entanto, as demais espécies avaliadas também apresentaram intensidades de defeitos aceitáveis, sugerindo que podem ser utilizadas na produção de madeira serrada, em regiões de ocorrência de geadas.
\end{abstract}

Palavras-chave: Desdobro da madeira; rachaduras; empenamentos.

\section{Lumber quality of five frost-resistant Eucalyptus species}

\begin{abstract}
Eucalyptus cultivation in the South Brazil is still limited due to the occurrence of frost and the limited number of species adapted to this condition. So, this study aimed to evaluate the wood quality of five Eucalyptus species cultivated in a frost occurrence region. Eucalyptus benthamii, Eucalyptus deanei, Eucalyptus dorrigoensis, Eucalyptus dunnii and Eucalyptus smithii trees, 18 years old, were sectioned into logs, which were divided into diametric grades of (1) 20,1 to $25,0 \mathrm{~cm}$; (2) 25,1 to $30,0 \mathrm{~cm}$ and (3) greater than $30,0 \mathrm{~cm}$. All the logs were submitted to the sawing, producing boards with tangential faces. Before and after the natural drying, the intensities of splits and warping (spring, bow and cupping) of the boards were determined. The results indicated that the log diameter had little influence on the lumber quality. The drying process increased splits and spring intensities, without tendency for bow intensity. The lowest defect intensities were presented by Eucalyptus dunnii and Eucalyptus deanei lumber. However, the other evaluated species also showed acceptable defect intensities, suggesting that it can be used for lumber production, in frost occurrence region.
\end{abstract}

Keywords: Sawing wood; splits; warping. 


\section{Introdução}

O sul do Brasil apresenta grande potencial florestal, destacando-se como o maior produtor de florestas plantadas de pinus do país. Contudo, nos últimos anos, tem se verificado a estagnação e até mesmo a redução da área cultivada com este gênero na região, o que decorre, principalmente, da substituição do cultivo do pinus pelo eucalipto, a exemplo do que vem acontecendo em outras regiões do Brasil. Dados da Indústria Brasileira de Árvores (IBÁ) (2017), indicam que nos últimos cinco anos a área plantada com pinus no país apresentou uma redução na ordem de $0,7 \%$ a.a.

Essa substituição e, consequentemente, o aumento do cultivo de Eucalyptus estão relacionados às características silviculturais que o gênero apresenta, sobretudo as elevadas taxas de crescimento e a maior resistência as condições edafoclimáticas (TRUGILHO et al., 2004) e vêm sendo verificado, inclusive, em localidades da região sul sujeitas a ocorrência de geadas, porém de forma limitada, uma vez que o número de espécies que se adaptam a essa condição climática é restrito (BRONDANI et al., 2010).

Atualmente, nestes locais, destacam-se como principais espécies cultivadas o Eucalyptus dunnii e o Eucalyptus benthamii, que foram introduzidos visando atender as necessidades da indústria de celulose e papel. No entanto, outras espécies, como Eucalyptus deanei (HIGA et al., 1997), Eucalyptus dorrigoensis (SIMÃO et al., 2011) e Eucalyptus smithii (FRIGOTTO et al., 2015), também vêm apresentando desenvolvimento silvicultural satisfatório em plantios experimentais, podendo, num futuro breve, vir a substituir outras espécies tradicionalmente utilizadas pela indústria florestal local.

Porém, para que isso seja possível se fazem necessários estudos que avaliem o potencial de utilização dessas espécies, em especial, na produção de serrados. De acordo com
Ferreira et al. (2004), quando empregada com esta finalidade, a madeira de eucalipto tende a apresentar alguns inconvenientes como a formação de rachaduras e empenamentos (arqueamento, encurvamento e encanoamento). Segundo Calonego; Severo (2005), esses defeitos podem se manifestar durante os processos de desdobro e secagem e são ocasionados principalmente pelas tensões internas de crescimento que em algumas situações podem inviabilizar o uso de determinadas espécies.

Frequentemente, alguns autores (CALONEGO; SEVERO, 2005; LIMA et al., 2006; CADEMARTORI et al., 2015) costumam relacionar a intensidade das manifestações das tensões de crescimento às características silviculturais, ou de exploração, mas especialmente às características da própria espécie e/ou árvore, com destaque para o diâmetro de toras. No entanto, não há um consenso quanto ao efeito do diâmetro de toras na manifestação dessas tensões, uma vez que na literatura são encontradas diferentes correlações.

Carvalho et al. (2010), ao estudarem a madeira de Corymbia citriodora e Eucalyptus urophylla, verificaram que a redução das tensões de crescimento estava associada ao incremento da classe diamétrica, sugerindo a adoção do diâmetro de toras como parâmetro de seleção de indivíduos com menores intensidades de tensões de crescimento. Enquanto Lima; Garcia (2008) ao avaliarem a madeira de Eucalyptus grandis, adotando as rachaduras de extremidades das tábuas como meio indireto para determinação das tensões de crescimento, verificaram a elevação dessas tensões com o aumento do diâmetro. Por outro lado, Trugilho et al. (2007) ao estudarem as tensões de crescimento da madeira de clones de Eucalyptus, não verificaram correlação entre essas características.

As deformações decorrentes das tensões de crescimento também são observadas durante o processo de secagem da madeira e podem ser acentuadas devido ao efeito combinado 
das tensões de crescimento com as tensões de secagem. Stangerlin et al. (2009) ao estudarem os efeitos da secagem na qualidade da madeira de Corymbia citriodora, Eucalyptus saligna e Eucalyptus tereticornis, verificaram a elevação dos índices de rachaduras e intensidades de arqueamento e a redução das intensidades de encurvamento não observando a manifestação de encanoamento. O mesmo foi observado por Rocha; Tomaselli (2002) para a madeira de Eucalyptus grandis e Eucalyptus dunnii, diferindo apenas com relação aos resultados de encanoamento, defeito este que passou a ser observado para o Eucalyptus dunnii após o processo de secagem.

Diante dos aspectos levantados, esse estudo teve como objetivo avaliar a qualidade da madeira serrada de cinco espécies de Eucalyptus cultivadas em região de ocorrência de geadas levando em consideração a classe diamétrica das toras e o processo de secagem da madeira.

\section{Material e Métodos}

As árvores foram coletadas em povoamentos experimentais de Eucalyptus benthamii, Eucalyptus deanei, Eucalyptus dorrigoensis, Eucalyptus dunnii e Eucalyptus smithii com 18 anos de idade. O material foi plantado no espaçamento de 3x2 m, na Fazenda Triângulo (26²9’37” S e 4942’32” W), interior de Mafra/SC. A região caracteriza-se por apresentar precipitação total anual de 1360 a $1670 \mathrm{~mm} \mathrm{e}$ temperatura média anual entre 15,5 e $17^{\circ} \mathrm{C}$, com temperatura média máxima entre 26 e $24^{\circ} \mathrm{C}$ e temperatura média mínima entre 10,8 e 11,8 ${ }^{\circ} \mathrm{C}$. De acordo com a classificação de Köppen, o clima é do tipo temperado, constantemente úmido, sem estação seca, com verão fresco (Cfb) (EPAGRI/CIRAM, 2016).

Para cada espécie foram selecionadas seis árvores, fora da área de bordadura e que apresentavam bom aspecto fitossanitário, tronco cilíndrico e sem bifurcações e diâmetro a 1,30 m do solo (D1,30m) de 30 a $40 \mathrm{~cm}$. Após a derrubada, as árvores foram traçadas em toras com $2,10 \mathrm{~m}$ de comprimento. No total, foram selecionadas 75 toras, sendo 15 por espécie, divididas em três classes diamétricas (classe 1: 20,1 a 25,0 cm; classe 2: 25,1 a 30,0 cm; classe 3: maior que $30,0 \mathrm{~cm})$, resultando em cinco toras por tratamento.

No processo de desdobro, todas as toras produziram tábuas com faces tangenciais e espessura nominal de $21 \mathrm{~mm}$, sem desprezar a região da medula. Sendo que foram utilizados três modelos de corte gerados pelo programa MaxiTora. O modelo 1 (Figura 1) foi empregado no processamento das toras pertencentes à classe 1 , em que as toras passaram por três vezes em serra fita simples vertical e posteriormente foram resserradas em cortes sucessivos paralelos, com a mesma serra, obtendo seis tábuas com largura de $115 \mathrm{~mm}$. As quatro costaneiras foram reaproveitadas em serra fita simples horizontal e refiladas em serra circular múltipla de cinco discos, resultando em tábuas com larguras de $50 \mathrm{~mm}$ (uma peça), $75 \mathrm{~mm}$ (uma peça) e $85 \mathrm{~mm}$ (duas peças).

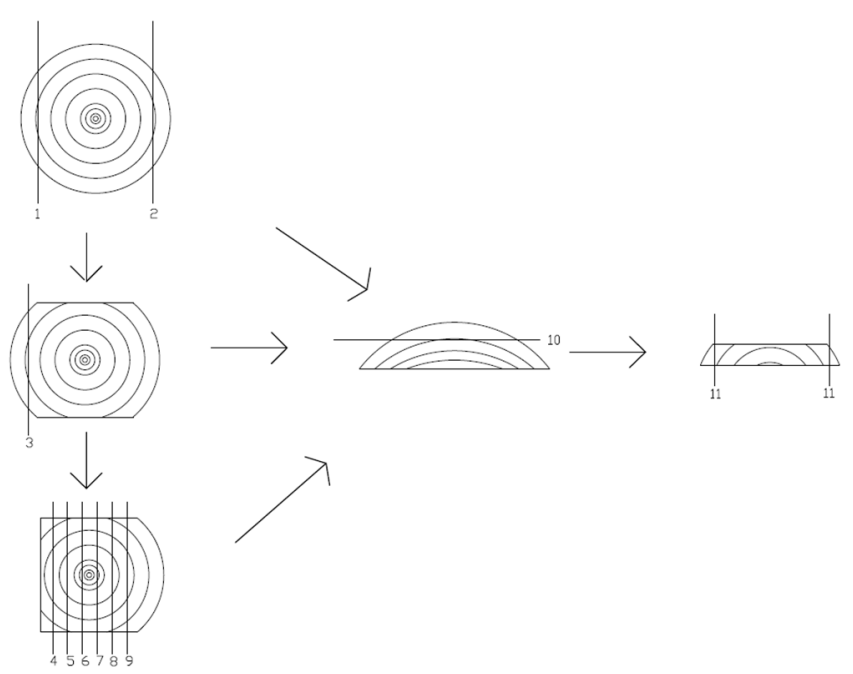

Figura 1. Descrição do processo de desdobro do modelo 1. Figure 1. Sawn pattern \#1 description of the sawmilling process.

O modelo 2, utilizado no desdobro das toras da classe 2, foi muito semelhante ao modelo 1, diferindo em relação a 
este, no número de tábuas originadas no desdobro do bloco com face curva (oito peças) e na largura das tábuas geradas no reaproveitamento das costaneiras, que foi de $75 \mathrm{~mm}$ (duas peças) e $130 \mathrm{~mm}$ (duas peças) (Figura 2).

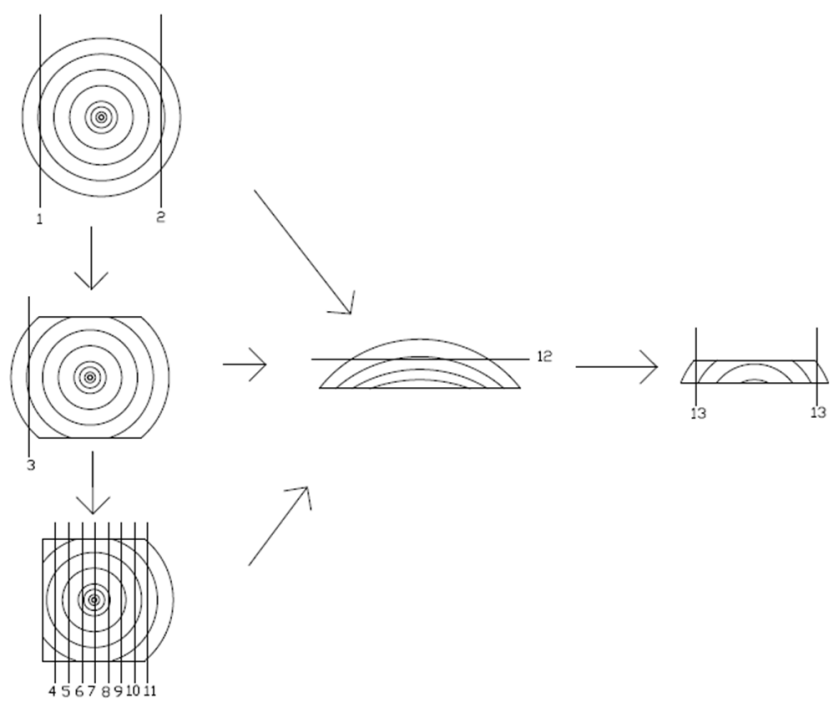

Figura 2. Descrição do processo de desdobro do modelo 2.

Figure 2. Sawn pattern \#2 description of the sawmilling process.

O modelo 3 (Figura 3) foi empregado no processamento das toras da classe 3. Neste modelo, as toras passaram duas vezes na serra fita simples vertical para a formação do semibloco que foi resserrado, produzindo nove tábuas de 105 mm de largura e duas costaneiras que foram descartadas. Já as outras duas costaneiras, obtidas na fase inicial, passaram três vezes em serra fita simples horizontal e posteriormente foram refiladas em serra circular múltipla de cinco discos, produzindo tábuas com larguras de $105 \mathrm{~mm}$ (quatro peças), $130 \mathrm{~mm}$ (duas peças) e $110 \mathrm{~mm}$ (duas peças) (Figura 3).

Como parâmetros de qualidade foram considerados o índice de rachaduras e as intensidades de empenamentos (arqueamento, encurvamento e encanoamento). Estes parâmetros foram determinados de acordo com a metodologia descrita por Rocha; Trugilho (2006), ponderando duas condições de umidade, uma logo após o desdobro (condição saturada) e outra após o processo de secagem (condição seca), avaliando todas as tábuas produzidas.

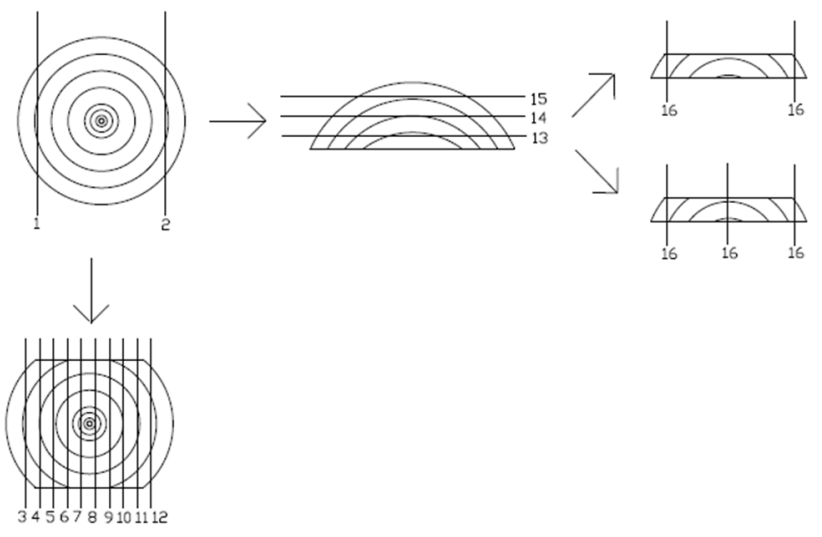

Figura 3. Descrição do processo de desdobro do modelo 3.

Figure 3. Sawn pattern \#3 description of the sawmilling process.

O processo de secagem foi conduzido naturalmente no pátio da serraria em que foi realizado o desdobro das toras, no distrito de Bateias de Cima, município de Campo Alegre, Estado de Santa Catarina. Para tanto, as tábuas foram distribuídas, aleatoriamente, em quatro pilhas de secagem, cobertas por um período de cinco meses até atingirem 17,5\% de umidade média. Na montagem das pilhas, cada uma das camadas de tábuas foi distribuída sobre cinco tabiques de dimensões de $2,5 \times 2,5 \times 120 \mathrm{~cm}$, distantes $42 \mathrm{~cm}$ entre si.

A análise estatística foi realizada em delineamento inteiramente casualizado, em arranjo fatorial ( $5 \times 3 \times 2)$, considerando o nível de 5\% de probabilidade de erro. Os fatores considerados foram espécie (cinco níveis), classe diamétrica (três níveis) e condição de umidade (dois níveis).

Os dados foram testados quanto à normalidade, por meio do teste de Shapiro Wilk, e quanto à homogeneidade das variâncias, por meio do teste de Levene. Quando não verificados estes pressupostos, foram realizadas transformações matemáticas utilizando-se funções de logaritmo neperiano, raiz quadrada e transformação de BoxCox para atender os critérios da estatística paramétrica. 
Posteriormente, os resultados foram avaliados por meio de análise de variância (ANOVA) e sempre que rejeitada a hipótese de nulidade $(\mathrm{p}<0,05)$, realizou-se o teste de Tukey para comparação de médias.

\section{Resultados e Discussão}

Quando considerados os resultados da análise de variância (Tabela 1), verifica-se que a espécie apresentou efeito significativo sobre todos os parâmetros de qualidade avaliados, enquanto o efeito da classe diamétrica foi observado para o índice de rachaduras e intensidades de arqueamento e encurvamento.

Quanto ao efeito da condição de umidade, o mesmo não foi considerado para o encanoamento, visto que este defeito não é observado em tábuas na condição saturada, já que tem origem no processo de secagem da madeira, devido à diferença de retratibilidade entre as faces radial e tangencial da tábua. Assim, considerando os demais defeitos este foi observado de forma significativa nos resultados de índice de rachaduras e intensidade de arqueamento, não interferindo nos resultados de encurvamento.
Sobre a interação espécie $\mathrm{x}$ classe diamétrica, a mesma apresentou efeito significativo sobre todos os parâmetros estudados, destacando-se, em conjunto com o fator espécie, como as fontes de variação de maior influência na qualidade da madeira serrada.

Para a interação espécie $\mathrm{x}$ condição de umidade, seu efeito só foi verificado para o encurvamento. Já as interações classe diamétrica $\mathrm{x}$ condição de umidade e espécie $\mathrm{x}$ classe diamétrica $\mathrm{x}$ condição de umidade apresentaram efeito significativo sobre o arqueamento e encurvamento, não sendo observado para o índice de rachaduras.

\section{Rachaduras}

De acordo com os resultados do teste de médias para o índice de rachaduras (Tabela 2), ainda que a análise de variância tenha apontado o efeito significativo da classe diamétrica, não foram observadas diferenças para esta variável quando analisados os resultados individuais por espécie, avaliando a mesma condição de umidade, indicando que este efeito somente ocorreu quando considerados os resultados obtidos entre espécies e condições de umidade distintas, ou ainda, para a média geral das classes diamétricas.

Tabela 1. Análise de variância para os resultados dos parâmetros de qualidade.

Table 1. Analysis of variance for the quality parameters results.

\begin{tabular}{|c|c|c|c|c|}
\hline \multirow{2}{*}{ Fontes de variação } & \multicolumn{4}{|c|}{ Valores de $\mathrm{P}$} \\
\hline & IR & A & Ev & Ec \\
\hline Espécie & $2,48^{\mathrm{e}-06^{*}}$ & $5,73^{\mathrm{e}-08^{*}}$ & $6,24^{\mathrm{e}-08^{*}}$ & $1,88^{\mathrm{e}-07^{*}}$ \\
\hline Classe diamétrica & $0,01^{*}$ & $6,87^{\mathrm{e}-10^{*}}$ & $<2,20^{\mathrm{e}-16^{*}}$ & $0,28^{\mathrm{ns}}$ \\
\hline Condição de umidade & $1,75^{\mathrm{e}-10^{*}}$ & $2,10^{\mathrm{e}-10^{*}}$ & $0,69^{\text {ns }}$ & --- \\
\hline Espécie x Classe diamétrica & $1,45^{\mathrm{e}-05^{*}}$ & $<2,20^{\mathrm{e}-16^{*}}$ & $<2,20^{\mathrm{e}-16^{*}}$ & $0,00^{*}$ \\
\hline Espécie x Condição de umidade & $0,84^{\mathrm{ns}}$ & $0,01^{\mathrm{ns}}$ & $9,09^{\mathrm{e}-06^{*}}$ & -- \\
\hline Classe diamétrica x Condição de umidade & $0,92^{\text {ns }}$ & $2,55^{\mathrm{e}-07^{*}}$ & $2,58^{\mathrm{e}-06^{*}}$ & $-\cdots--$ \\
\hline Espécie x Classe diamétrica x Condição de umidade & $0,96^{\mathrm{ns}}$ & $2,30^{\mathrm{e}-09^{*}}$ & $<2,20^{\mathrm{e}-16^{*}}$ & ------. \\
\hline
\end{tabular}

${ }^{*}$ Significativo a $5 \%$ de probabilidade $(\mathrm{p}<0,05)$, ${ }^{\mathrm{ns}}$ não significativo $(\mathrm{p}>=0,05)$. Em que: $\mathrm{IR}=$ índice de rachaduras; $\mathrm{A}=$ intensidade de arqueamento; $\mathrm{Ev}=$ intensidade de encurvamento e $\mathrm{Ec}=$ intensidade de encanoamento. 
Tabela 2. Índices de rachaduras médios para madeira serrada em diferentes condições de umidade.

Table 2. Mean splits rates for lumber in different humidity conditions.

\begin{tabular}{|c|c|c|c|c|c|}
\hline \multicolumn{6}{|c|}{ Índice de rachaduras (\%) } \\
\hline $\begin{array}{l}\text { Condição de } \\
\text { umidade }\end{array}$ & Espécie & Classe 1 & Classe 2 & Classe 3 & Média \\
\hline \multirow{5}{*}{ Saturada } & E. benthamii & $\begin{array}{c}11,91 \mathrm{AB} \mathrm{a} \\
(78,33)\end{array}$ & $\begin{array}{c}13,57^{\mathrm{AB} a} \\
(94,19)\end{array}$ & $\begin{array}{l}11,02^{\mathrm{AB} \mathrm{a}} \\
(107,82)\end{array}$ & $\begin{array}{c}12,09 \text { ABCD } \\
(96,35)\end{array}$ \\
\hline & E. deanei & $\begin{array}{l}12,11 \mathrm{AB} \text { a } \\
(107,73)\end{array}$ & $\begin{array}{c}6,36^{\text {В а }} \\
(152,50)\end{array}$ & $\begin{array}{c}6,25^{\text {Ва }} \\
(146,32)\end{array}$ & $\begin{array}{c}7,58^{\mathrm{D}} \\
(138,76)\end{array}$ \\
\hline & E. dorrigoensis & $\begin{array}{l}7,51 \mathrm{AB} \mathrm{a} \\
(95,43)\end{array}$ & $\begin{array}{c}12,544^{\mathrm{AB} a} \\
(82,18)\end{array}$ & $\begin{array}{c}6,40^{\mathrm{B} \mathrm{а}} \\
(182,62)\end{array}$ & $\begin{array}{c}8,34^{\mathrm{CD}} \\
(134,29)\end{array}$ \\
\hline & E. dunnii & $\begin{array}{c}5,15^{\text {В а }} \\
(109,64)\end{array}$ & $\begin{array}{l}9,05^{\text {В а }} \\
(97,12)\end{array}$ & $\begin{array}{l}9,97 \mathrm{AB} \text { a } \\
(173,25)\end{array}$ & $\begin{array}{l}8,59^{\mathrm{CD}} \\
(152,92)\end{array}$ \\
\hline & E. smithii & $\begin{array}{l}10,29 \mathrm{AB} \text { a } \\
(72,17)\end{array}$ & $\begin{array}{c}13,60 \mathrm{AB} \text { a } \\
(65,37)\end{array}$ & $\begin{array}{c}11,31 \mathrm{AB} a \\
(89,00)\end{array}$ & $\begin{array}{c}11,73^{\mathrm{BCD}} \\
(79,00)\end{array}$ \\
\hline \multirow{5}{*}{$\begin{array}{c}\text { Secagem natural a } \\
\qquad 17,5 \%\end{array}$} & E. benthamii & $\begin{array}{l}15,25^{\mathrm{AB} a} \\
(106,27)\end{array}$ & $\begin{array}{l}19,18^{\text {A a }} \\
(109,12)\end{array}$ & $\begin{array}{l}13,56^{\mathrm{AB} \mathrm{a}} \\
(123,93)\end{array}$ & $\begin{array}{l}15,84^{\mathrm{AB}} \\
(115,10)\end{array}$ \\
\hline & E. deanei & $\begin{array}{l}19,20^{\text {A a }} \\
(134,75)\end{array}$ & $\begin{array}{l}10,92 \mathrm{AB} a \\
(183,92)\end{array}$ & $\begin{array}{l}10,11 \mathrm{AB} \mathrm{a} \\
(186,55)\end{array}$ & $\begin{array}{l}12,37 \mathrm{ABC} \\
(171,19)\end{array}$ \\
\hline & E. dorrigoensis & $\begin{array}{l}9,90 \mathrm{AB} \text { a } \\
(95,43)\end{array}$ & $\begin{array}{c}14,56^{\mathrm{AB} a} \\
(82,18)\end{array}$ & $\begin{array}{l}10,11^{\mathrm{AB} a} \\
(182,62)\end{array}$ & $\begin{array}{l}11,28^{\text {BCD }} \\
(134,29)\end{array}$ \\
\hline & E. dunnii & $\begin{array}{l}9,94^{\mathrm{AB} \mathrm{a}} \\
(153,01)\end{array}$ & $\begin{array}{l}13,27 \mathrm{AB} \mathrm{a} \\
(112,35)\end{array}$ & $\begin{array}{l}15,68^{\mathrm{A} \mathrm{a}} \\
(164,79)\end{array}$ & $\begin{array}{c}13,63^{\mathrm{AB}} \\
(152,782)\end{array}$ \\
\hline & E. smithii & $\begin{array}{l}13,80^{\mathrm{AB} a} \\
(111,68)\end{array}$ & $\begin{array}{l}20,29^{\mathrm{A} \mathrm{a}} \\
(110,28)\end{array}$ & $\begin{array}{l}15,46^{\mathrm{Aa}} \\
(126,93)\end{array}$ & $\begin{array}{c}16,45^{\mathrm{A}} \\
(119,77)\end{array}$ \\
\hline
\end{tabular}

Médias seguidas de mesma letra, maiúscula na coluna e minúscula na linha, não diferem estatisticamente (Tukey, $\mathrm{p}>0,05)$; valores entre parênteses referem-se ao coeficiente de variação das amostras.

Deste modo, não foi possível a determinação de um comportamento para a classe diamétrica. Entretanto, destacam-se os resultados da classe 2, que para o Eucalyptus dorrigoensis, Eucalyptus benthamii e Eucalyptus smithii, nas diferentes condições de umidade, apresentaram numericamente as maiores intensidades deste defeito em comparação às demais classes. Possivelmente, este resultado está relacionado ao maior índice de rachaduras de topo das toras apresentado pelo material selecionado para compor esta classe, conforme o observado durante o processo de avaliação da qualidade das toras, cujos resultados foram apresentados em outro trabalho.

Outra causa provável foi a largura das tábuas, já que o modelo de desdobro utilizado na classe 2, no geral, produziu tábuas mais largas em relação às produzidas nas outras classes. Se consideradas as dimensões e o número de tábuas produzidas em cada modelo de desdobro, verifica-se que para classe 2 a largura média das tábuas foi de 110,83 mm, enquanto para as classes 1 e 3 , foi de $93,08 \mathrm{~mm}$ e $108,53 \mathrm{~mm}$, respectivamente. Assim, em função dos modelos de desdobro adotados, constatou-se que as tábuas mais largas foram retiradas da região mais interna do bloco, que normalmente está submetida aos maiores esforços de tensão, em comparação as demais regiões. Além disso, durante a avaliação da madeira serrada, verificou-se que as tábuas retiradas dessas regiões mais internas e que continham medula, considerando as tábuas sem a presença dessa característica anatômica, apresentavam maiores índices de rachaduras. Crêspo (2000), ao estudar as tensões de crescimento e os seus efeitos sobre o desdobro e a secagem de Eucalyptus grandis e Eucalyptus saligna, mas sem justificar os resultados, observou uma tendência semelhante, verificando 
que as rachaduras das tábuas aumentaram proporcionalmente à sua largura.

Em relação à condição de umidade, ainda que se observe a elevação dos índices de rachaduras após o processo de secagem para todos os tratamentos avaliados, somente foram observadas diferenças significativas para as médias gerais obtidas para Eucalyptus deanei, Eucalyptus smithii e Eucalyptus dunnii. De tal modo, foi observado que E. dunnii apresentou proporcionalmente maior aumento da intensidade deste defeito, indicando uma maior instabilidade dimensional em comparação às demais espécies.

Resultados análogos foram obtidos por Rocha; Trugilho (2006) que ao avaliarem os defeitos da madeira serrada de Eucalyptus dunnii, verificaram o aumento de $37 \%$ no valor das rachaduras após o processo de secagem, concluindo que este defeito é influenciado, não só pelas tensões internas de crescimento, como também pelas tensões de secagem. Todavia Eleotério et al. (2014) e Souza et al. (2012) ao avaliarem outras espécies do mesmo gênero, considerando os índices de rachaduras médios, não observaram diferença significativa entre condições de umidade distintas.

Quanto ao fator espécie, foram observadas diferenças significativas apenas após o processo de secagem para as médias gerais, verificando-se as maiores intensidades de rachaduras para o Eucalyptus smithii e o Eucalyptus benthamii e as menores intensidades para o Eucalyptus dorrigoensis, seguido pelo Eucalyptus deanei e Eucalyptus dunnii. Uma das possíveis causas deste resultado, da mesma forma ao observado para o efeito do diâmetro, foram as rachaduras de topo das toras, uma vez que as maiores intensidades deste defeito foram observadas nas toras de Eucalyptus smithii, enquanto as menores nas toras de Eucalyptus dorrigoensis. Lima et al. (2002) ao avaliarem metodologias de determinação das rachaduras de topo das toras e a sua relação com as rachaduras na madeira serrada de
Eucalyptus grandis, verificaram que as rachaduras de topo das toras respondiam por até $50 \%$ das rachaduras das extremidades das peças serradas.

No geral, exceto para Eucalyptus deanei, Eucalyptus dorrigoensis e Eucalyptus dunnii após o desdobro, os índices de rachaduras observados foram maiores do que o padrão estabelecido pela Norma para a Classificação de Madeira Serrada de Folhosas (INSTITUTO BRASILEIRO DE DESENVOLVIMENTO FLORESTAL, 1983), que determina o limite de até $10 \%$ para enquadramento na primeira classe. Contudo, estão de acordo com os valores observados para o gênero Eucalyptus por autores que adotaram a mesma metodologia de avaliação e não excluem a possibilidade de utilização dessas espécies como matéria-prima para a produção de madeira serrada. Hornburg et al. (2012), por exemplo, ao estudarem a madeira de seis espécies de eucalipto na condição saturada, incluindo espécies já utilizadas para produção de serrados como Eucalyptus grandis e Eucalyptus cloeziana, verificaram índices de rachaduras médios para todas as espécies variando entre 2,54\% e 12,28\%. Caixeta et al. (2002), ao avaliarem a madeira de híbridos de Eucalyptus spp. após o processo de secagem, observaram o índice de rachaduras médio na ordem de 16,06\%. Já Cademartori et al. (2015), estudando a madeira de Eucalyptus grandis, verificaram índices de rachaduras após o desdobro variando entre 5,17 e 9,61\%, e após a secagem entre 8,26\% e 11,31\%.

Os resultados de intensidade de arqueamento (Tabela 3) indicaram que o efeito do diâmetro variou de acordo com a espécie e a condição de umidade. Assim, para a condição saturada foram observadas diferenças significativas entre as classes diamétricas somente para os valores obtidos por Eucalyptus dorrigoensis e Eucalyptus smithii, verificando-se as maiores intensidades de arqueamento na classe de maior diâmetro. Logo, para a condição seca a 17,5 \% de umidade, todas as espécies, exceto Eucalyptus dunnii, apresentaram 
diferenças significativas entre as classes diamétricas. Contudo, como nessa condição as classes que apresentaram as maiores ou menores intensidades de arqueamento variaram de espécie para espécie não foi possível a determinação de uma tendência quanto ao diâmetro de toras.

Essas diferenças sobre o efeito do diâmetro das toras nos resultados de intensidade de arqueamento podem estar relacionadas às características de algumas toras selecionadas neste estudo, como o deslocamento de medula (LIMA et al., 2007). Durante o desdobro da tora o deslocamento da medula pode causar o encurvamento do semibloco que é convertido em arqueamento quando este é processado em tábuas (SOUZA et al., 2012). Assim, tábuas provenientes de toras com deslocamento de medula tendem a apresentar maior arqueamento do que as tábuas obtidas a partir de toras sem esta característica.

Do mesmo modo ao observado para o efeito do diâmetro, o efeito da secagem sobre a intensidade de arqueamento também variou entre os tratamentos. Porém, quando consideradas as médias gerais obtidas por espécie, verifica-se que as intensidades de arqueamento para Eucalyptus benthamii, Eucalyptus dorrigoensis e Eucalyptus smithii aumentaram significativamente com o processo de secagem, enquanto para Eucalyptus dunnii não foram notadas variações.

Tabela 3. Arqueamento na madeira serrada em diferentes condições de umidade.

Table 3. Spring in lumber in different humidity conditions.

\begin{tabular}{|c|c|c|c|c|c|}
\hline \multicolumn{6}{|c|}{ Intensidade de arqueamento $(\mathrm{mm} / \mathrm{m})$} \\
\hline $\begin{array}{l}\text { Condição de } \\
\text { umidade }\end{array}$ & Espécie & Classe 1 & Classe 2 & Classe 3 & Média \\
\hline \multirow{10}{*}{ Saturada } & \multirow{2}{*}{ E. benthamii } & $1,57 \mathrm{CDE}$ a & $0,92^{\text {В а }}$ & $1,75^{\mathrm{Ca}}$ & $1,43^{\mathrm{D}}$ \\
\hline & & $(138,06)$ & $(153,94)$ & $(92,91)$ & $(121,75)$ \\
\hline & \multirow{2}{*}{ E. deanei } & $0,98^{\mathrm{DE} a}$ & $2,28 \mathrm{AB} \mathrm{a}$ & $1,94^{\mathrm{BC} \text { a }}$ & $1,83^{\mathrm{CD}}$ \\
\hline & & $(127,97)$ & $(111,94)$ & $(74,28)$ & $(102,20)$ \\
\hline & \multirow{2}{*}{ E. dorrigoensis } & $0,41^{\mathrm{Eb}}$ & $1,38^{\text {B ab }}$ & $2,23 \mathrm{BC}$ a & $1,56^{\mathrm{D}}$ \\
\hline & & $(183,13)$ & $(76,22)$ & $(84,93)$ & $(105,36)$ \\
\hline & \multirow{2}{*}{ E. dunnii } & $0,88^{\mathrm{DE} a}$ & $1,86^{\mathrm{AB} \text { a }}$ & $2,21 \mathrm{BCa}$ & $1,80^{\mathrm{CD}}$ \\
\hline & & $(168,10)$ & $(67,77)$ & $(88,38)$ & $(96,51)$ \\
\hline & \multirow{2}{*}{ E. smithii } & $2,16^{\mathrm{BCD} \text { ab }}$ & $1,01^{\mathrm{B}}$ & $3,12^{\mathrm{AB} \text { a }}$ & $2,32^{\mathrm{BC}}$ \\
\hline & & $(83,27)$ & $(136,67)$ & $(85,21)$ & $(101,64)$ \\
\hline \multirow{10}{*}{$\begin{array}{c}\text { Secagem natural a } \\
17,5 \%\end{array}$} & \multirow{2}{*}{ E. benthamii } & $3,31^{\mathrm{ABC} a}$ & $2,22^{\mathrm{AB} a b}$ & $1,68^{\mathrm{Cb}}$ & $2,25^{\mathrm{BC}}$ \\
\hline & & $(83,72)$ & $(138,58)$ & $(138,32)$ & $(122,77)$ \\
\hline & \multirow{2}{*}{ E. deanei } & $2,09^{\mathrm{BCDE} a b}$ & $1,45^{\mathrm{B} \mathrm{b}}$ & $2,95 \mathrm{AB}$ a & $2,31^{\mathrm{BC}}$ \\
\hline & & $(109,88)$ & $(134,86)$ & $(98,60)$ & $(112,48)$ \\
\hline & \multirow{2}{*}{ E. dorrigoensis } & $3,88^{\mathrm{A} \mathrm{a}}$ & $3,21^{\mathrm{Aab}}$ & $1,87^{\mathrm{Cb}}$ & $2,72^{\mathrm{AB}}$ \\
\hline & & $(60,81)$ & $(75,45)$ & $(141,70)$ & $(97,78)$ \\
\hline & \multirow{2}{*}{ E. dunnii } & $1,06^{\mathrm{DE} \mathrm{a}}$ & $1,90^{\mathrm{AB} a}$ & $2,07^{\mathrm{BC} \mathrm{a}}$ & $1,80^{\mathrm{CD}}$ \\
\hline & & $(170,48)$ & $(111,80)$ & $(118,58)$ & $(125,82)$ \\
\hline & \multirow{2}{*}{ E. smithii } & $3,79 \mathrm{AB}$ a & $0,75^{\text {в b }}$ & $4,04^{\mathrm{A} \mathrm{a}}$ & $3,06^{\mathrm{A}}$ \\
\hline & & $(83,20)$ & $(314,99)$ & $(104,79)$ & $(125,19)$ \\
\hline
\end{tabular}

Médias seguidas de mesma letra, maiúscula na coluna e minúscula na linha, não diferem estatisticamente (Tukey, $\mathrm{p}>0,05)$; valores entre parênteses referem-se ao coeficiente de variação das amostras. 
Esse aumento no arqueamento após o processo de secagem, está de acordo com resultados encontrados por Cademartori et al. (2015) e assim como observado para o índice de rachaduras, indica que além das tensões de crescimento, o arqueamento também está relacionado às tensões de secagem. Neste sentido, Stangerlin et al. (2009) destacam que na secagem da madeira o arqueamento apresenta-se como um dos defeitos de difícil controle, o que decorre da organização das tábuas nas pilhas, que por não apresentarem nenhuma restrição lateral não impedem as deformações das tábuas.

Sobre a influência das espécies nos resultados, verificouse que aquelas a se destacarem com os maiores e menores intensidade de arqueamento, variaram de classe para classe e condição de umidade. Todavia, na condição saturada os resultados médios finais indicaram que significativamente as maiores intensidades de arqueamento foram apresentadas pelo Eucalyptus smithii e as menores pelo Eucalyptus benthamii e Eucalyptus dorrigoensis. Já na condição seca, as maiores intensidades de arqueamento foram apresentadas novamente pelo Eucalyptus smithii e as menores para Eucalyptus dunnii, seguido pelo Eucalyptus benthamii e Eucalyptus deanei. Provavelmente estes resultados estão relacionados às rachaduras das tábuas, em especial no caso do Eucalyptus smithii que foi uma das espécies a apresentar os maiores índices de rachaduras.

Vázquez (2001) ao descrever o conjunto de forças que se manifestam na liberação das tensões de crescimento durante o desdobro das toras ressalta que o arqueamento das tábuas está relacionado com as rachaduras, uma vez que a formação dos dois defeitos decorre dos esforços de tensão perpendicular que se originam no centro das tábuas.

Quando consideradas as intensidades de arqueamento médias obtidas para todos os tratamentos, nota-se que os valores apresentados por todas as espécies estão dentro dos limites estabelecidos pela norma ABNT NBR 14806 (2002), que determina como intensidade máxima de arqueamento para a madeira serrada de eucalipto o valor de até $5 \mathrm{~mm} / \mathrm{m}$ no caso de tábuas a serem incluídas na primeira classe. Esses resultados se caracterizam como um bom indicativo da qualidade da madeira serrada das espécies estudadas e se aproximam dos obtidos por outros autores ao estudarem o gênero Eucalyptus. Anjos; Fonte (2017), ao avaliarem a intensidade de arqueamento da madeira úmida de Eucalyptus grandis, Eucalyptus dunnii e Eucalyptus saligna, observaram valores entre 2,13 e 2,69 mm/m. Enquanto Eleotério et al. (2014), ao estudarem a qualidade da madeira serrada de seis espécies de eucalipto, verificaram intensidades entre 1,80 e 3,80 $\mathrm{mm} / \mathrm{m}$ após o desdobro, e entre 1,90 e 6,60 mm/m após a secagem.

Para o efeito das classes diamétricas, os resultados do teste de médias para a intensidade de encurvamento (Tabela 4) apontaram diferenças nos casos de E. dorrigoensis, na condição saturada e E. benthamii, E. dunnii e E. smithii, na condição seca, verificando-se as menores intensidades do defeito para a classe de maior diâmetro.

Com relação aos demais tratamentos, numericamente, também foram observadas as menores intensidades de encurvamento na classe 3. Estes resultados se aproximam dos observados por Rocha; Tomaselli (2002), que para Eucalyptus grandis e Eucalyptus dunnii, observaram que, no geral, as menores intensidades de encurvamento foram apresentadas pela classe de maior diâmetro. Resultados similares foram obtidos por Anjos; Fonte (2017), que embora tenham concluído que a classe diamétrica exerceu pouca influência sobre o encurvamento, verificaram uma tendência de redução da intensidade desse defeito com a elevação da classe diamétrica. 
Tabela 4. Encurvamento na madeira serrada em diferentes condições de umidade.

Table 4. Bow in lumber in different humidity conditions.

\begin{tabular}{|c|c|c|c|c|c|}
\hline \multicolumn{6}{|c|}{ Intensidade de encurvamento $(\mathrm{mm} / \mathrm{m})$} \\
\hline $\begin{array}{c}\text { Condição de } \\
\text { umidade }\end{array}$ & Espécie & Classe 1 & Classe 2 & Classe 3 & Média \\
\hline \multirow{5}{*}{ Saturada } & E. benthamii & $\begin{array}{c}3,20^{\mathrm{BCD} a} \\
(55,15)\end{array}$ & $\begin{array}{l}2,23^{\mathrm{BC} a} \\
(80,89)\end{array}$ & $\begin{array}{l}2,03^{\mathrm{AB} a} \\
(51,79)\end{array}$ & $\begin{array}{l}2,37^{\mathrm{BCD}} \\
(66,48)\end{array}$ \\
\hline & E. deanei & $\begin{array}{l}2,50 \mathrm{CD} \mathrm{a} \\
(86,17)\end{array}$ & $\begin{array}{l}2,72^{\mathrm{BC} \text { a }} \\
(56,33)\end{array}$ & $\begin{array}{l}1,83^{\mathrm{AB} a} \\
(81,94)\end{array}$ & $\begin{array}{l}2,25^{\mathrm{BCD}} \\
(76,27)\end{array}$ \\
\hline & E. dorrigoensis & $\begin{array}{l}1,90^{\mathrm{Dab}} \\
(88,22)\end{array}$ & $\begin{array}{l}3,25^{\text {В а }} \\
(85,20)\end{array}$ & $\begin{array}{l}1,50^{\mathrm{B} \mathrm{b}} \\
(93,81)\end{array}$ & $\begin{array}{l}2,04 \mathrm{CD} \\
(99,42)\end{array}$ \\
\hline & E. dunnii & $\begin{array}{l}3,78^{\mathrm{BC} \text { a }} \\
(66,19)\end{array}$ & $\begin{array}{l}3,14^{\text {В а }} \\
(65,67)\end{array}$ & $\begin{array}{l}2,55^{\mathrm{A} \mathrm{a}} \\
(80,96)\end{array}$ & $\begin{array}{c}3,01^{\mathrm{A}} \\
(73,62)\end{array}$ \\
\hline & E. smithii & $\begin{array}{l}3,43^{\mathrm{BC} \text { a }} \\
(56,79)\end{array}$ & $\begin{array}{l}3,09 \text { в а } \\
(60,50)\end{array}$ & $\begin{array}{l}2,17^{\mathrm{AB} \mathrm{a}} \\
(82,06)\end{array}$ & $\begin{array}{l}2,69 \mathrm{AB} \\
(71,07)\end{array}$ \\
\hline \multirow{5}{*}{$\begin{array}{c}\text { Secagem natural a } \\
17,5 \%\end{array}$} & E. benthamii & $\begin{array}{l}4,26^{\mathrm{AB} a} \\
(53,76)\end{array}$ & $\begin{array}{l}2,99^{\text {В а }} \\
(90,15)\end{array}$ & $\begin{array}{l}1,49^{\mathrm{BCb}} \\
(103,18)\end{array}$ & $\begin{array}{l}2,76^{\mathrm{AB}} \\
(89,93)\end{array}$ \\
\hline & E. deanei & $\begin{array}{l}1,71^{\mathrm{Da}} \\
(131,63)\end{array}$ & $\begin{array}{l}1,60 \mathrm{CD} \mathrm{a} \\
(124,66)\end{array}$ & $\begin{array}{l}1,76^{\mathrm{AB} a} \\
(119,12)\end{array}$ & $\begin{array}{c}1,70^{\mathrm{D}} \\
(123,04)\end{array}$ \\
\hline & E. dorrigoensis & $\begin{array}{l}5,30^{\mathrm{A} \mathrm{a}} \\
(39,29)\end{array}$ & $\begin{array}{l}4,78^{\mathrm{A} \mathrm{a}} \\
(54,35)\end{array}$ & $\begin{array}{c}0,45^{\mathrm{Cb}} \\
(259,24)\end{array}$ & $\begin{array}{c}2,73^{\mathrm{AB}} \\
(108,26)\end{array}$ \\
\hline & E. dunnii & $\begin{array}{c}2,91 \text { BCD ab } \\
(89,63)\end{array}$ & $\begin{array}{l}3,35^{\text {В а }} \\
(65,51)\end{array}$ & $\begin{array}{l}1,99 \text { АВ } \\
(90,41)\end{array}$ & $\begin{array}{l}2,61 \text { ABC } \\
(84,25)\end{array}$ \\
\hline & E. smithii & $\begin{array}{l}4,37 \mathrm{AB} \mathrm{a} \\
(67,19)\end{array}$ & $\begin{array}{l}0,65^{\mathrm{Dc}} \\
(217,65)\end{array}$ & $\begin{array}{l}2,63 \mathrm{Ab} \\
(98,01)\end{array}$ & $\begin{array}{l}2,44^{\mathrm{ABC}} \\
(111,63)\end{array}$ \\
\hline
\end{tabular}

Médias seguidas de mesma letra, maiúscula na coluna e minúscula na linha, não diferem estatisticamente (Tukey, $\mathrm{p}>0,05)$; valores entre parênteses referem-se ao coeficiente de variação das amostras.

Sobre a decorrência da secagem na intensidade de encurvamento, apesar deste processo normalmente reduzir as intensidades deste defeito (CADEMARTORI et al., 2015), os diferentes tratamentos apresentaram disposições distintas. Assim, foram observadas diferenças significativas apenas para Eucalyptus dorrigoensis nas classes 1 e 2 que indicaram a elevação da intensidade de encurvamento após o processo de secagem e para Eucalyptus smithii na classe 2 que apresentou redução da intensidade do encurvamento após esse processo.

Porém, quando considerados os resultados médios por espécies, verifica-se que a secagem reduziu as intensidades deste defeito nas tábuas de Eucalyptus deanei, Eucalyptus dunnii e Eucalyptus smithii e aumentou, além das tábuas de Eucalyptus dorrigoensis, nas tábuas de Eucalyptus benthamii. Resultados semelhantes foram obtidos por Stangerlin et al.
(2009), que ao avaliarem o efeito de dois métodos de secagem na qualidade da madeira serrada de três espécies de eucalipto, verificaram que as flechas de encurvamento de todas as espécies estudadas demonstraram uma tendência de redução após a secagem, exceto na secagem em estufa solar de Eucalyptus saligna e na secagem ao ar livre de Corymbia citriodora. A mesma tendência foi observada por Eleotério et al. (2014), que ao estudarem o efeito de diferentes métodos na secagem da madeira de seis espécies de eucalipto, verificaram aumento na intensidade deste defeito somente na madeira de Eucalyptus cloeziana e Eucalyptus grandis submetidas a secagem a taxas moderadas.

No geral, os resultados aqui obtidos indicam que o peso das tábuas dispostas nas pilhas não foi suficiente para restringir mecanicamente a elevação do encurvamento 
durante a secagem da madeira. Contudo, essa proposição não é suficiente para justificar a elevação considerável das intensidades de encurvamento observadas para Eucalyptus dorrigoensis, uma vez que as tábuas foram organizadas aleatoriamente em diferentes pilhas de secagem, evitando-se também a concentração de tábuas por espécie em uma única região da pilha. Os resultados obtidos sugerem que são provocados por uma combinação de fatores, que incluem além das condições de secagem, as propriedades intrínsecas à madeira, como a instabilidade dimensional.

Na comparação entre espécies, aquelas que se destacaram significativamente com as maiores e menores intensidades de encurvamento, variaram entre as classes diamétricas e condições de umidade, indicando que o resultado observado para cada tratamento foi mais influenciado pela interação dos fatores avaliados do que pelo efeito isolado do fator espécie. Porém, analisando os valores médios obtidos por espécie, verifica-se que, na condição saturada as menores flechas de encurvamento foram apresentadas pela madeira de Eucalyptus dorrigoensis e as maiores pelas madeiras de Eucalyptus dunnii e Eucalyptus smithii. Enquanto que para a condição seca, as menores flechas foram observadas para Eucalyptus deanei e as maiores para Eucalyptus benthamii e Eucalyptus dorrigoensis

As intensidades de encurvamento observadas para todas as espécies, nos diferentes tratamentos avaliados, apresentam valores médios inferiores a $5 \mathrm{~mm} / \mathrm{m}$, atendendo os limites estabelecidos pela norma ABNT NBR 14806 (2002) para a inclusão da madeira serrada na Primeira Classe. Além disso, estão de acordo com os valores observados para a madeira de eucalipto. Cademartori et al. (2015), ao avaliarem a madeira de Eucalyptus grandis, observaram intensidade de encurvamento entre 4,52 e $6,17 \mathrm{~mm} / \mathrm{m}$ após o desdobro e entre 4,17 e 5,22 mm/m após a secagem. Enquanto, Stangerlin et al. (2009) verificaram valores entre 1,49 e 2,08, após o desdobro, e entre 1,50 a 2,03 $\mathrm{mm} / \mathrm{m}$, após a secagem para a madeira de Corymbia citriodora, Eucalyptus saligna e Eucalyptus tereticornis, respectivamente.

Para o encanoamento, os resultados (Tabela 5) apontaram diferenças entre as classes diamétricas apenas para Eucalyptus dorrigoensis, observando-se a redução da intensidade deste defeito com o incremento do diâmetro de toras, o que, considerando a análise de variância foi provocado pela interação dos fatores espécie e classe diamétrica. Para as demais espécies, exceto Eucalyptus benthamii, verifica-se que, numericamente, as classes 1 e 2 destacaram-se com as maiores intensidades.

Sobre o efeito da espécie na intensidade de encanoamento, verificam-se diferenças significativas nas classes 1 e 2 e na média geral, observando-se, nestas três situações as maiores intensidades para a madeira de Eucalyptus dorrigoensis e as menores para a madeira de Eucalyptus dunnii e Eucalyptus deanei. Estes resultados, assim como observado sobre o efeito da classe diamétrica, podem estar relacionados à instabilidade dimensional da madeira.

As intensidades de encanoamento obtidas por todas as espécies nas diferentes condições avaliadas foram menores do que as observadas por outros autores ao estudarem a madeira de Eucalyptus, adotando a mesma metodologia de avaliação. Calonego; Severo (2007) observaram valores entre 1,21 e 4,41 mm para Eucalyptus grandis. Já Rocha; Trugilho (2006) verificaram uma intensidade de encanoamento média de 2,88 mm para Eucalyptus dunnii. No entanto, deve-se destacar que alguns autores, como Stangerlin et al. (2009) e Cademartori et al. (2015) ao realizarem estudos semelhantes não verificaram a ocorrência de encanoamento, atribuindo os seus resultados ao correto empilhamento da madeira adotado para a condução do processo de secagem. 
Tabela 5. Encanoamento na madeira serrada.

Table 5. Cupping in lumber.

\begin{tabular}{|c|c|c|c|c|c|}
\hline \multicolumn{6}{|c|}{ Intensidade de encanoamento $(\mathrm{mm})$} \\
\hline $\begin{array}{l}\text { Condição de } \\
\text { umidade }\end{array}$ & Espécie & Classe 1 & Classe 2 & Classe 3 & Média \\
\hline \multirow{5}{*}{$\begin{array}{c}\text { Secagem natural a } \\
17,5 \%\end{array}$} & E. benthamii & $\begin{array}{c}0,33^{\mathrm{Ba}} \\
(305,27)\end{array}$ & $\begin{array}{c}0,53^{\mathrm{B} \mathrm{a}} \\
(262,04)\end{array}$ & $\begin{array}{l}0,72^{\mathrm{A} \mathrm{a}} \\
(151,43)\end{array}$ & $\begin{array}{c}0,56^{\mathrm{BC}} \\
(210,50)\end{array}$ \\
\hline & E. deanei & $\begin{array}{l}0,80^{\mathrm{AB} \mathrm{a}} \\
(185,79)\end{array}$ & $\begin{array}{c}0,56^{\text {в а }} \\
(182,24)\end{array}$ & $\begin{array}{l}0,73^{\mathrm{A} \mathrm{a}} \\
(169,88)\end{array}$ & $\begin{array}{c}0,69^{\mathrm{AB}} \\
(178,21)\end{array}$ \\
\hline & E. dorrigoensis & $\begin{array}{l}1,41^{\mathrm{A} \mathrm{a}} \\
(133,78)\end{array}$ & $\begin{array}{l}1,34^{\mathrm{Aa}} \\
(135,28)\end{array}$ & $\begin{array}{c}0,63 \mathrm{Ab} \\
(176,48)\end{array}$ & $\begin{array}{c}1,00^{\mathrm{A}} \\
(155,73)\end{array}$ \\
\hline & E. dunnii & $\begin{array}{c}0,20^{\mathrm{B} \mathrm{a}} \\
(264,24)\end{array}$ & $\begin{array}{c}0,46^{\text {В а }} \\
(181,72)\end{array}$ & $\begin{array}{c}0,25^{\mathrm{A} \mathrm{a}} \\
(274,29)\end{array}$ & $\begin{array}{c}0,31^{\mathrm{C}} \\
(234,18)\end{array}$ \\
\hline & E. smithii & $\begin{array}{l}0,65^{\mathrm{AB} a} \\
(228,86)\end{array}$ & $\begin{array}{l}0,59 \mathrm{AB} \text { a } \\
(219,57)\end{array}$ & $\begin{array}{c}0,55^{\mathrm{A} \mathrm{a}} \\
(200,11)\end{array}$ & $\begin{array}{c}0,58^{\mathrm{BC}} \\
(213,25)\end{array}$ \\
\hline
\end{tabular}

Médias seguidas de mesma letra, maiúscula na coluna e minúscula na linha, não diferem estatisticamente (Tukey, $p>0,05$ ); valores entre parênteses referem-se ao coeficiente de variação das amostras.

\section{Conclusões}

Dentre os fatores avaliados, a espécie e a interação espécie $\mathrm{x}$ classe diamétrica destacaram-se como os de maior influência na qualidade de madeira serrada.

A classe diamétrica apresentou pouca influência sobre os parâmetros de qualidade, verificando-se apenas no caso do encurvamento tendência de redução da intensidade deste defeito com a elevação da classe diamétrica.

$\mathrm{O}$ processo de secagem da madeira aumentou as intensidades de rachadura e arqueamento.

As menores intensidades de defeitos, especialmente após a secagem, foram apresentadas pela madeira de Eucalyptus dunnii e Eucalyptus deanei.

Todas as espécies avaliadas, nas diferentes condições de umidade, apresentaram intensidades de defeitos compatíveis com valores encontrados na literatura para espécies de Eucalyptus já empregadas na produção de serrados, sugerindo que estas podem ser empregadas com a mesma finalidade em regiões de ocorrência de geadas.

\section{Referências}

ANJOS, R. A. M.; FONTE, A. P. N. Comparação da qualidade da madeira serrada de três espécies de Eucalyptus. Ambiência, Guarapuava, v. 13, n. 1, p. 151 - 165, 2017.

ASSOCIAÇÃO BRASILEIRA DE NORMAS TÉCNICAS. NBR 14806: Madeira serrada de eucalipto - Requisitos. Rio de Janeiro. 2002.

BRONDANI, E. G.; WENDLING, I.; GROSSI, F.; DUTRA, L. F.; ARAUJO, M. A. Miniestaquia de Eucalyptus benthamii x Eucalyptus dunnii: (II) Sobrevivência e enraizamento de miniestacas em função das coletas e estações do ano. Ciência Florestal, Santa Maria, v. 20, n. 3, p. 453 - 465, 2010.

CADEMARTORI, P. H. G.; GATTO, D. A.; STANGERLIN, D. M.; MASTELLA, T.; VARGAS, J. F. V.; SANTINI, E. J. Uso de herbicida na redução das tensões de crescimento na madeira serrada de Eucalyptus grandis. Ciência Florestal, Santa Maria, v. 25, n. 3, p. 801 - 808, 2015.

CALONEGO, F. W.; SEVERO, E. T. D. Efeito do diâmetro de toras na magnitude das tensões de crescimento de Eucalyptus grandis. Energia na Agricultura, Botucatu, v. 20, n. 2, p. 5365,2005 .

CALONEGO, F. W.; SEVERO, E. T. D. Influência da posição diametral das tábuas e da vaporização nos defeitos de secagem de Eucalyptus grandis. Cerne, Lavras, v. 13, n. 1, p. 10-18, 2007. 
CARVALHO, A. M.; GONÇALVES, M. P. M.; AMPARADO, K. F.; LATORRACA, J. V. F.; GARCIA, R. A. Correlações da altura e diâmetro com tensões de crescimento em árvores de Corymbia citriodora e Eucalyptus urophylla. Revista Árvore, Viçosa, v. 34, n. 2, p. 323 - 331, 2010.

ELEOTÉRIO, J. R.; HORNBURG, K. F.; REICHERT, D.; BAGATTOLI, T. R.; MENEGHELLI, I. Efeito da espécie e da condição de secagem na formação de defeitos na madeira serrada de eucalipto. Scientia Forestalis, Piracicaba, v. 42, n. 101, p. 41 - 47, 2014.

\section{EPAGRI/CIRAM. Zoneamento agroecológico e} socioeconômico do Estado de Santa Catarina. Disponível em:

<http://www.ciram.epagri.sc.gov.br/images/documentos/Zo nAgroecoMapas.pdf >. Acessado em 07/12/2016.

FERREIRA, S.; LIMA, J. T.; ROSADO, S. C. S.; TRUGILHO, P. F. Influência de métodos de desdobro tangenciais no rendimento e na qualidade da madeira de clones de Eucalyptus spp. Cerne, Lavras, v. 10, n. 1, p. 10-21, 2004.

FRIGOTTO, T.; NAVROSKI, M. C.; PEREIRA, M. O.; MAZZO, M. V.; SOUZA, P. F. Seleção de procedências de Eucalyptus smithii na região norte de Santa Catarina. In: CONGRESSO BRASILEIRO DE EUCALIPTO, III., 2015, Vitória. Anais... Vitória: [S. I.], 2015. p. [S. I.].

HIGA, R.C.V.; HIGA, A.R.; TREVISAN, R.; SOUZA, M.V.R. Comportamento de 20 espécies de Eucalyptus em área de ocorrência de geadas na região sul do Brasil. In: IUFRO CONFERENCE ON SILVICULTURE AND IMPROVEMENT OF Eucalyptus, 1997, Salvador. Anais... Colombo: EMBRAPA-CNPF, 1997. p. 106 - 110.

HORNBURG, K. F.; ELEOTÉRIO, J. E.; BAGATTOLI, T. R.; NICOLETTI, A. L. Qualidade das toras e da madeira serrada de seis espécies de eucalipto cultivadas no litoral de Santa Catarina. Scientia Forestalis, Piracicaba, v. 40, n. 96, p. 463471,2012

\section{INDÚSTRIA BRASILEIRA DE ÁRVORES (IBÁ). Relatório

2017. Disponível em: <
http://iba.org/images/shared/Biblioteca/IBA_RelatorioAnua 12017.pdf>. Acessado em: 7 set. 2017.

INSTITUTO BRASILEIRO DE DESENVOLVIMENTO FLORESTAL. Norma para a classificação de madeira serrada de folhosas. Brasília. 1983.
LIMA, I. L.; GARCIA, J. N. Influência do desbaste e da fertilização nos índices de rachamento após o desdobro e secagem em madeira serrada de Eucalyptus grandis Hill exMaiden. Revista do Instituto Florestal, São Paulo, v. 20, n. 2, p. $175-184,2008$.

LIMA, I. L.; GARCIA, J. N.; PIEDADE, S. M. S. Rachaduras de extremidades de tora e suas implicações nas rachaduras da madeira serrada. Scientia Forestalis, Piracicaba, n. 61, p. 13 24, 2002.

LIMA, I. L.; GARCIA, J. N.; STAPE, J. L.; PIEDADE, S. M. S. Efeito do desbaste e da fertilização nas tensões de crescimento em Eucalyptus grandis. Scientia Forestalis, Piracicaba, n. 70, p. $171-183,2006$.

LIMA, I. L.; GARCIA, J. N.; STAPE, J. L. Influência do desbaste e da fertilização no deslocamento da medula e rachaduras de extremidade de tora de Eucalyptus grandis Hill ex-Maiden. Cerne, Lavras, v. 13, n. 2, p. 170-177, 2007.

ROCHA, M. P.; TOMASELLI, I. Efeito do modelo de desdobro na qualidade da madeira serrada de Eucalyptus grandis e Eucalyptus dunnii. Cerne, Lavras, v. 8, n. 2, p. 7083, 2002.

ROCHA, M. P.; TRUGILHO, P. F. Qualidade da madeira serrada de Eucalyptus dunnii em função do método de desdobro e condição de umidade. Cerne, Lavras, v. 12, n. 4, p. 314-321, 2006.

SIMÃO, R. L.; NARCISO, A. G. S. O.; SOMMER, S. M. Characteristics of Wood and Pulp from Eucalyptus dorrigoensis Trees. In: INTERNATIONAL COLLOQUIUM ON Eucalyptus PULP, 5., 2011, Porto Seguro. Anais... Porto Seguro: [S. I.], 2011. p. [S. I.].

SOUZA, J. T.; TREVISAN, R.; DENARDI, L.; STANGERLIN, D. M.; VIVIAN, M. A.; HASELEIN, C. R.; SANTINI, E. J. Qualidade da Madeira serrada proveniente de árvores dominantes e médias de Eucalyptus grandis submetidas à secagem. Cerne, Lavras, v. 18, n. 1, p. 167-174, 2012.

STANGERLIN, D. M.; SANTINI, E. J.; SUSIN, F., MELO, R. R.; GATTO, D. A.; HASELEIN, C. R. Uso de estufa solar para secagem de madeira serrada. Ciência Florestal, Santa Maria, v. 19, n. 4, p. 461 - 472, 2009.

TRUGILHO, P. F.; IWAKIRI, S.; ROCHA, M. P.; MATOS, J. L. M.; SALDANHA, L. K.

Efeitos da idade e classe diamétrica na deformação residual longitudinal em árvores 
de Eucalyptus dunnii Maiden. Árvore, Viçosa, v. 28, n. 1, p. 38-44, 2004.

TRUGILHO, P. F.; ROSADO, S. C. S.; LIMA, J. T.; PÁDUA, F. A.; SOUZA, M. A. M. Deformação residual longitudinal (DRL) e sua relação com as características de crescimento da árvore em clones de Eucalyptus. Cerne, Lavras, v. 13, n. 2, p. 130 - 137, 2007.

VÁZQUEZ, M. C. T. Tensiones de crescimento em Eucalyptus globulus de Galicia (España): Influencia de la silvicultura y estrategias de aserrado. Maderas. Ciencia y tecnologia, Concepción, v. 3, n. 1-2, p. 68 - 89, 2001. 